\title{
Alveolar soft part sarcoma associated with lung and brain metastases: A case report
}

\author{
MINGGUANG WANG ${ }^{1}$, JINXING LI $^{1}$, LINCHUN HUAN ${ }^{1}$, FANGUO MENG $^{1}$ and QI PANG $^{2}$ \\ ${ }^{1}$ Department of Neurosurgery, People's Hospital of Linyi City of Shandong Province, Linyi, Shandong 276003; \\ ${ }^{2}$ Department of Neurosurgery, Provincial Hospital of Medical College of Shandong University, \\ Jinan, Shandong 250000, P.R. China
}

Received November 19, 2014; Accepted July 28, 2016

DOI: $10.3892 / \mathrm{ol} .2016 .4715$

\begin{abstract}
The present case report aimed to improve the understanding of alveolar soft part sarcoma (ASPS) by investigating the clinical characteristics, diagnosis and therapeutic methods used to treat ASPS associated with lung and brain metastases. The clinical data of a single patient diagnosed with ASPS by postoperative pathology was studied retrospectively, and additional associated reports and previous studies of similar cases were reviewed. Clinical symptoms were markedly alleviated following surgical treatment, followed by a chemotherapy regime. During post-treatment follow-up, no tumor recurrence was observed.
\end{abstract}

\section{Introduction}

Alveolar soft part sarcoma (ASPS) is a clinically rare soft tissue sarcoma (STS) of unknown histological origin, accounting for $0.5-1.0 \%$ of all STS cases, worldwide (1). ASPS associated with lung and brain metastases is a rare disease with poor prognosis, with a high risk of metastasis to the brain. Surgery is typically the primary method used in the treatment of ASPS (2). The present study outlines the case of a patient with ASPS and associated lung and brain metastases. The patient provided signed informed consent, and therefore the case is detailed in the following report, including the course of disease and patient outcome.

\section{Case report}

A 39-year-old male patient was admitted to the People's Hospital of Linyi City of Shandong Province (Linyi, China) on 3 January 2014, after experiencing occasional headaches

Correspondence to: Mr. Mingguang Wang, Department of Neurosurgery, People's Hospital of Linyi City of Shandong Province, 27 Jiefang Road, Linyi, Shandong 276003, P.R. China

E-mail:w1975mg@sohu.com

Key words: alveolar soft part sarcoma metastasis, brain metastases, treatment during the previous 6 months. The patient's headaches had become progressively more severe 1 month prior to admission to hospital, and were associated with right upper limb weakness and inaccurate fine motor movements. Physical examination revealed negative cranial nerve-associated signs. Breath sounds were clear in both lungs, and no rhonchi or moist rales were heard. Distal right upper limb muscle strength received grade 4 (3), and tendon reflex and pathological signs were negative. As revealed by cranial computed tomography (CT; Fig. 1A), a high-density lesion was observed in the left postfrontal area [CT values, 52 Hounsfield units, (HU)] and a peripheral low-density edematous zone was visible. Magnetic resonance imaging (MRI) was also performed and revealed an irregular lump in the left frontal lobe with T1 and T2 hyperintensity. The surrounding vessels were belt-like and anfractuous with no intensified edematous regions (Fig. 1B-D). The medical history of the patient included resection of a subcutaneous mass in the left lower limb 10 years previously, which recurred 5 years later and required a second surgery. The masses resected during these earlier surgeries had not been sent for pathological examination. The patient reported no family history of the manifestations described above. Subsequently, the patient was further examined using chest radiography, which indicated multiple pulmonary nodules on both lungs. Therefore, the patient underwent single-photon emission CT/CT, which revealed no significant abnormalities on a whole-body bone scan (Fig. 1E). The chest CT result (Fig. 1F) supported the prior chest radiography result. Laboratory examinations revealed that the tuberculin skin test was negative and the erythrocyte sedimentation rate was $3.6 \mathrm{mmol} / \mathrm{min}$. Therefore, the patient was diagnosed with a metastatic tumor and underwent surgical resection. During surgery, a purplish-red tumor was observed in the brain. The tumor adhered closely to the dura mater and was solid, with an abundant blood supply. A number of dilated vessels had developed on the tumor surface and these were connected with veins on the surface of the brain. Following complete resection, histopathological examination of a hematoxylin and eosin-stained section via microscopy at 10x20 magnification revealed a diagnosis of ASPS (Fig. 1G). The tumor cells were arranged in an alveolar manner and a few vascular fibrous tissues were distributed among them. The tumor cells appeared larger with mild nuclear pleomorphism and prominent nucleoli. The cells 

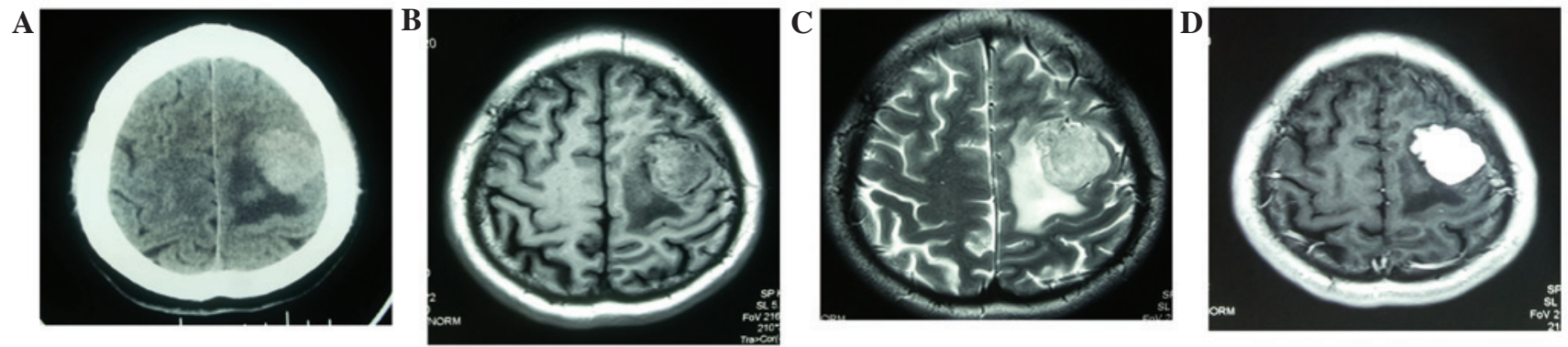

$\mathbf{E}$

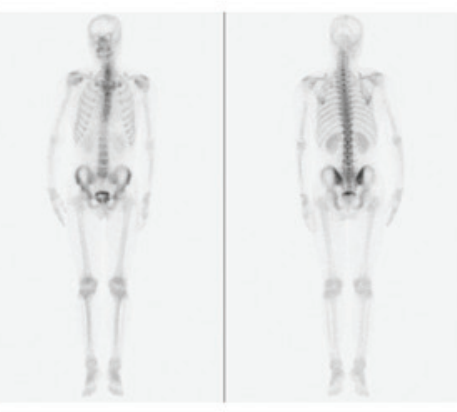

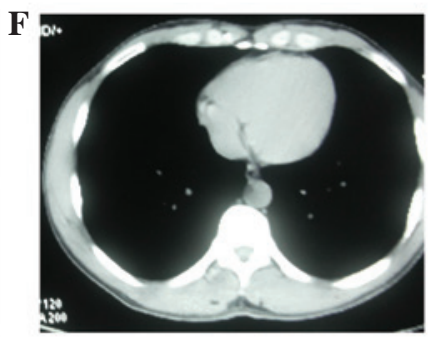

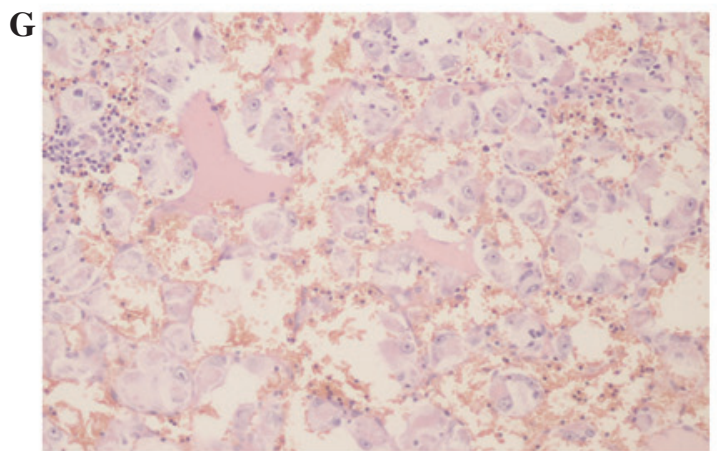

Figure 1. CT, MRI and pathological examination. (A) Cranial CT revealed a high-density lesion in the left postfrontal area and a low-density shadow in the surrounding tissues. (B-D) MRI indicated an irregular lump in the left frontal lobe with T1 and T2 hyperintensity and marked enhancement. (E) Anterior (left) and posterior (right) single photon emission CT/CT revealed no significant abnormalities on a whole-body bone scan. (F) Chest CT indicated multiple pulmonary nodules on each lung. (G) Pathological analysis revealed tumor cells with prominent nucleoli, and the cytoplasm stained red and appeared transparent. Thus, the pathological diagnosis was alveolar soft part sarcoma (magnification, 10x20; stain, hematoxylin and eosin). CT, computed tomography; MRI, magnetic resonance imaging.
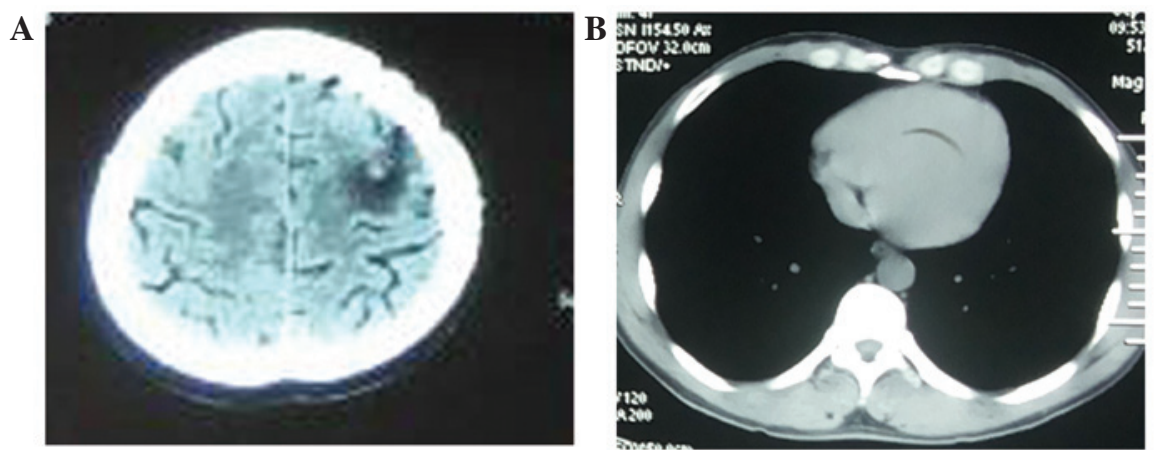

Figure 2. Follow-up CT scans performed 8 months after surgery. (A) Follow-up cranial CT scan revealed no intracranial tumor recurrence. (B) Chest CT indicated that the chest lesions were stable. CT, computed tomography.

were polygonal, round and cylindrical in shape. The cytoplasm was stained red and appeared transparent and a number of small granular pigments were identified within the cells. In addition, certain cells were arranged in distinct solid nests. The results of immunohistochemical analysis indicated positivity for actin, desmin, S-100 and periodic acid-Schiff stain, with negativity for cytokeratin, synaptophysin and chromogranin A. The postoperative right upper muscle strength score was 100 according to the Karnofsky performance status scoring system (4). Following surgery, the patient received 30 radiotherapy doses at 60 Gy per dose and was administered 4 courses of doxorubicin $\left(50 \mathrm{mg} / \mathrm{m}^{2}\right.$, every 3 weeks) and ifosfamide $\left(1.5 \mathrm{mg} / \mathrm{m}^{2}\right.$, every 4 weeks). Subsequently, CT and MRI examinations during the following 8 months demonstrated no recurrence of the intracranial tumor (Fig. 2A) or progression of the chest lesions (Fig. 2B).

\section{Discussion}

STS accounts for $\sim 1 \%$ of all malignant tumors and ASPS is rarely reported in clinical settings, accounting for only $0.5-1.0 \%$ of STS cases, worldwide (1). ASPS occurs at all ages but is most prevalent in females aged 13-35 years (1). ASPS most commonly affects the soft tissues of the pelvic cavity and lower limbs; however, in children, common lesion sites include the head and neck (5). ASPS tumors frequently metastasize to the lungs, bones and brain. The origin of ASPS remains to be elucidated, although the majority of researchers have hypothesized that it is myogenic (6). The pathological manifestations of ASPS were initially described by Smetana and Scott, who observed the pulmonary alveolus-like arrangements of the tumor cells (7). It is known that, in ASPS, chromosome 17q25 contains 
abnormal structures, particularly the specific chromosome translocation $\mathrm{t}(\mathrm{x} ; 17)(\mathrm{p} 11.2 ; \mathrm{q} 25)(8)$, which results in the generation of a fusion gene called ASPL-TFE3. Based on this key finding, reverse transcription-polymerase chain reaction has been utilized to detect the messenger RNA expression of ASPL-TFE 3 and thus provide a reliable molecular criterion for the diagnosis of ASPS (9). Although ASPS may metastasize to the brain, it does not induce any specific symptoms or signs, and has therefore posed difficulties for clinical diagnosis through imaging examinations (10). In addition, to the best of our knowledge, no effective standard treatments for ASPS associated with brain metastasis have been developed to date. Therefore, patients with ASPS are mainly treated via surgical resection in order to improve intracranial hypertension and other symptoms (2). As ASPS tumors possess an abundant blood supply, en bloc rather than fractional resection should be performed during surgery. Surgery has been revealed to prolong patient survival time (11). However, whether postoperative radiotherapies are required remains to be elucidated. ASPS associated with brain metastasis has been observed to be insensitive to chemotherapeutic drugs, therefore no specific chemotherapeutic treatment regimens have been developed (12). Notably, the patient in the present case received 30 radiotherapy doses at 60 Gy per dose and was administered 4 courses of chemotherapy with doxorubicin and ifosfamide. According to previous prospective studies $(13,14)$, cediranib, a recently developed molecular-target drug, may effectively treat ASPS associated with brain metastasis with an overall remission rate of up to $35 \%$ and 24 -week control rate of $84 \%$, thus providing a novel clinical treatment option.

In conclusion, ASPS is a rare tumor which exhibits a different tumor biology compared with other soft tissue sarcomas. In this study, the case of ASPS with metastasis to both the lungs and the brain was presented. At present, the optimum therapeutic strategy for the disease remains unclear. Total surgical resection may be effective in improving patient survival and molecular-targeting drugs may effectively treat ASPS in the future.

\section{References}

1. Ogose A, Morita T, Hotta T, Kobayashi H, Otsuka H, Hirata Y and Yoshida, S: Brain metastases in musculoskeletal sarcomas. Jpn J Clin Oncol 29: 245-247, 1999.

2. Fox BD, Patel A, Suki D and Rao G: Surgical management of metastatic sarcoma to the brain. J Neurosurg 110: 181-186, 2009.

3. Robert Schwartzman (ed): Neurologic Examination. 1st edition.Wiley-Blackwell, Oxford, 2006.

4. Schag CC, Heinrich RL and Ganz PA: Karnofsky performance status revisited: Reliability, validity, and guidelines. J Clin Oncology 2: 187-193, 1984.

5. Hunter BC, Devaney KO, Ferlito A and Rinaldo A: Alveolar soft part sarcoma of the head and neck region. Ann Otol Rhinol Laryngol 107: 810-814, 1998.

6. Rosai J, Dias P, Parham DM, Shapiro DN and Houghton P: MyoD1 protein expression in alveolar soft part sarcoma as confirmatory evidence of its skeletal muscle nature. Am J Surg Pathol 15: 974-981, 1991

7. Smetana HF and Scott WF Jr: Malignant tumors of nonchromaffin paraganglia. Mil Surg 109: 330-349, 1951.

8. Uppal S, Aviv H, Patterson F, Cohen S, Benevia J, Aisner S and Hameed M: Alveolar soft part sarcoma - reciprocal translocation between chromosome 17q25 and Xp11. Report of a case with metastases at presentation and review of the literature. Acta Orthop Belg 69: 182-187, 2003.

9. Williams A, Bartle G, Sumathi VP, Meis JM, Mangham DC, Grimer RJ and Kindblom LG: Detection of ASPL/TFE3 fusion transcripts and the TFE3 antigen in formalin-fixed, paraffin-embedded tissue in a series of 18 cases of alveolar soft part sarcoma: Useful diagnostic tools in cases with unusual histological features. Virchows Arch 458: 291-300, 2011.

10. Perry JR, Bilbao JM:Metastatic alveolar soft part sarcoma presenting as a dural -based cerebral mass. Neurosurgery 1994,34:168-170.

11. Lewis AJ: Sarcoma metastatic to the brain. Cancer 61: 593-601, 1988.

12. Reichardt $P$, Lindner T, Pink D, Thuss-Patience PC, Kretzschmar A and Dörken B:Chemotherapy in alveolar soft part sarcomas: What do we know? Eur J Cancer 39: 1511-1516, 2003.

13. Kummar S, Allen D, Monks A, Polley EC, Hose CD, Ivy SP, Turkbey IB, Lawrence S, Kinders RJ, Choyke P, et al: Cediranib for metastatic alveolar soft part sarcoma. J Clin Oncol 31: 2296-2302, 2013.

14. Sleijfer S, Ballman K and Verweij J: The future of drug development? Seeking evidence of activity of novel drugs in small groups of patients. J Clin Oncol 31: 2246-2248, 2013. 